\title{
DISPOSITIF DE PHOTOTHÉRAPIE EN FIBRES OPTIQUES TISSÉES pour traiter la jaunisse du nourrisson
}

\author{
Laure PÉRUCHON \\ Cédric BROCHIER \\ Pierre SAINT GIRONS \\ ${ }^{1}$ Brochier Technologies, \\ 90 rue Frederic Fays, \\ 69100 Villeurbanne, France \\ ${ }^{2}$ Neomedlight, \\ 90 rue Frederic Fays, \\ 69100 Villeurbanne, France \\ laure.peruchon@ \\ brochiertechnologies.com
}

Dans le domaine de la santé, certaines indications thérapeutiques se traitent par la lumière et pourraient donc être traitées par le tissu lumineux en fibres optiques, telles que les dermatites, les mucites, la régénération des tissus / cicatrisation, la douleur, ainsi que la jaunisse du nourrisson. Chaque application nécessite une intensité et une gamme de longueur d'onde bien définies. Les sources classiquement utilisées sont les tubes fluorescents (lumière blanche) ou à vapeur de mercure (lumière ultraviolette), mais de plus en plus, les LEDs peu consommatrices d'énergie s'imposent en photothérapie. Les inconvénients de ces sources restent leur rigidité et le dégagement de chaleur. le cerveau, notamment en fonction de sa concentration et de paramètres environnementaux (âge post-natal, infection, taux d'albumine...).

Deux méthodes permettent de soigner l'ictère: l'exsanguino-transfusion et la photothérapie. L'exsanguinotransfusion consiste à remplacer une grande partie du sang du nourrisson, ce qui n'est pas sans risque de contamination. La photothérapie est une thérapeutique efficace de l'ictère. Les photons générés par la source lumineuse atteignent la surface cutanée exposée. Dans un spectre étroit, de 420 à 490 nm, les photons sont absorbés par la bilirubine située dans la peau. L'interaction entre la bilirubine et la lumière induit la formation de photo-produits facilement éliminables dans les urines. La photothérapie permet l'élimination dela bilirubine sans métabolisme hépatique.

Les facteurs de l'efficacité de la photothérapie sont:

- la distance entre la source et le patient, la surface du corps exposée,

l'intensité de l'irradiation qui doit être au minimum de $30 \mu \mathrm{W} / \mathrm{cm}^{2} / \mathrm{nm}$, la gamme de longueur d'onde centrée à $460 \mathrm{~nm}$. est aujourd'hui utilisé dans le traitement de la jaunisse du nourrisson par photothérapie et commercialisé par la société NeoMedLight, spin off de Brochier technologies.

La jaunisse du nourrisson est due à une hyperbilirubinémie, conséquence de l'adaptation post-natale du métabolisme de la bilirubine, se traduisant par une élimination insuffisante de la bilirubine produite. L'ictère est fréquent chez le nouveau-né, mais la bilirubine libre peut être toxique pour
Figure 1. Photo du pad double de photothérapie (bagpad). 
Figure 2. Photo du pad simple (nest pad).

\section{Les sourices lumineuses pour traiter la jaunisse du nournisson.}

Les tubes fluorescents bleus ont un spectre large contenant la gamme de longueur d'onde permettant de soigner l'ictère. Cependant, ils produisent de la chaleur, ce qui induit une perte de poids chez le nourrisson. De plus, la forte intensité lumineuse induit des risques de dommage rétinal. Afin d'obtenir l'irradiance optimale, il est nécessaire d'utiliser de nombreux tubes, à une distance de 15 à $20 \mathrm{~cm}$ du patient.

Les diodes électroluminescentes (LEDs) bleues ont un spectre étroit avec un maximum à $460 \mathrm{~nm}$. Il s'agit d'une lumière froide permettant d'éviter des altérations significatives en perte d'eau via l'épiderme. Le risque de dommage rétinal est toujours présent. L'irradiance est optimale car ciblée à la bonne longueur d'onde et très intense, mais l'émission des LEDs est très focalisée.

Le pad de fibres optiques connectées à des LEDs bleues est, dans le cas des premiers dispositifs existants, de faibles intensités d'irradiation $(20 \mu \mathrm{W} /$ $\mathrm{cm}^{2} / \mathrm{nm}$ ) et homogénéité. La technologie développée par Brochier technologies bénéficie d'un savoir-faire spécifique de traitement de surface des fibres optiques pour obtenir une émission latérale de lumière homogène. Cette innovation permet également d'atteindre de fortes intensités d'irradiation, supérieures à $50 \mu \mathrm{W} / \mathrm{cm}^{2} / \mathrm{nm}$. électricité ne sont présents au niveau de la zone lumineuse.

L'innovation textile dans le domaine de la photothérapie pour le traitement de la jaunisse du nourrisson porte sur deux solutions émettant de la lumière bleue: le sac de couchage (bag pad) et la couverture (nest pad). Dans les deux cas, le tissu lumineux est protégé par une enveloppe de plastique transparent en polyuréthane contact cutané facilement nettoyable et désinfectable. De plus, chaque système est utilisé avec un usage unique en non-tissé mono patient. L'usage unique apporte du confort au nourrisson et absorbe les flux. Dans le cas du sac de couchage, l'usage unique contient une attache large pour positionner au mieux l'enfant sur le pad.

Le sac de couchage est principalement utilisé pour les bébés à terme, alors que la couverture est dédiée aux prématurés. Chaque dispositif a ses avantages. Le sac de couchage permet d'éclairer le maximum de surface corporelle du nourrisson, l'enfant étant glissé à l'intérieur du dispositif, et donc d'avoir un traitement plus rapide ( $f$ gure 1). La couverture est dédiée aux prématurés, qui sont souvent monitorés et nécessitent d'avoir un visuel sur leur abdomen (figure 2). rage, la signalétique, la dépollution et la santé.

Le principe de la fibre optique est de conduire la lumière dans le tissu. Afin d'obtenir un éclairage latéral par les fibres optiques, celles-ci subissent un traitement de surface de microtexturation. L'utilisation de la fibre optique a également l'avantage de pouvoir déporter la source de lumière, car seule la zone ayant subi un traitement de surface devient lumineuse. Ainsi ni chaleur, ni

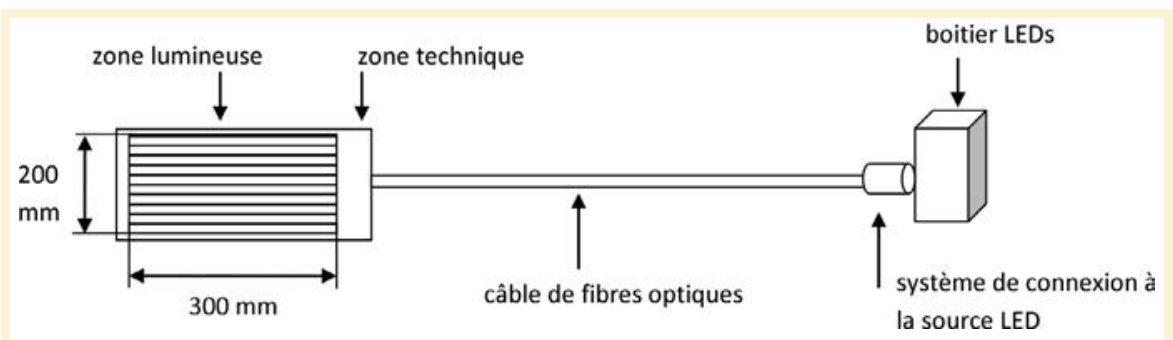

Figure 3. Schéma du pad de photothérapie. 
avantageusement les tests in vivo sur des populations de patients, qui ne sont pas faisables. Les paramètres pris en compte sont (i) la longueur d'onde du pic d'émission, (ii) la gamme spectrale d'émission, (iii) l'uniformité de l'intensité de l'irradiance, (iv) la surface du corps exposé et (v) l'âge de la source. Ces tests ont été menés par l'équipe du Docteur Cortey, responsable de l'unité clinique du Centre National de Référence en Hémobiologie Périnatale (CNRHP) à l'Hôpital Trousseau de Paris. L'efficacité des dispositifs médicaux repose sur le temps de demi-vie de la bilirubine non conjuguée. Plus le temps de demi-vie est court et plus la photothérapie est efficace. Une dizaine de dispositifs ont été étudiés et peuvent être répartis en trois catégories: les dispositifs à haute $\left(t_{1 / 2}<25 \mathrm{~min}\right.$, où $t_{1 / 2}$ est le temps de demi-vie), moyenne $\left(t_{1 / 2}<26-50 \mathrm{~min}\right)$ et faible $\left(\mathrm{t}_{1 / 2}>50 \mathrm{~min}\right)$ efficacité.

Les pads lumineux étudiés sont de format $100 \times 300 \mathrm{~mm}$ (figure 3). Le pad en simple tissu a une irradiance de 5 $\mathrm{mW} / \mathrm{cm}^{2}$ et se positionnera sous le nourrisson. Ce pad peut être recouvert d'un non-tissé à des fins d'hygiène. Dans ce cas-là, l'intensité de l'irradiance diminue à $3 \mathrm{~mW} / \mathrm{cm}^{2}$. Le second pad est en double tissu, soit deux tissus identiques superposés, et atteint $8 \mathrm{~mW} / \mathrm{cm}^{2}$. Ce pad très intense se place également sous le nourrisson. Enfin, il est toujours possible de mettre un pad sous le dos du nourrisson et un autre sur son ventre. Ainsi, la surface du corps éclairé est deux fois plus grande.

Les tests in vitro sont réalisés sur les 2 pads. Dans certains cas, le pad est testé recouvert d'un non-tissé et il peut également être plié en deux pour simuler un éclairage par le dessus et le dessous. Dans le cas du pad simple tissu (éclairage de dessous uniquement) à $5 \mathrm{~mW} / \mathrm{cm}^{2}$, le $\mathrm{t}_{1 / 2}$ obtenu est de $20 \mathrm{~min}$, tandis qu'à $3 \mathrm{~mW} / \mathrm{cm}^{2}$ (avec le non-tissé), le $t_{1 / 2}$ est de $30 \mathrm{~min}$. Enfin, si le pad éclaire dessus et dessous à $3 \mathrm{~mW} / \mathrm{cm}^{2}$, le $t_{1 / 2}$ n'est plus que de $7 \mathrm{~min}$. Dans le cas du pad à $8 \mathrm{~mW} / \mathrm{cm}^{2}$, le $\mathrm{t}_{1 / 2} \mathrm{ob}$ tenu est de $15 \mathrm{~min}$ pour un éclairage par-dessous et de $4 \mathrm{~min}$ pour un éclairage dessus et dessous. Ces résultats montrent l'intérêt de la technologie du textile lumineux. Celui-ci apporte souplesse et légèreté en comparaison au système rigide LED ou tube fluo tout en étant très performant.

Les conséquences d'une photothérapie trop intense, $\mathrm{t}_{1 / 2}<15 \mathrm{~min}$, sont méconnues et pas nécessaires. Aussi, le pad lumineux sélectionné suite à ces essais est le pad simple tissu à $5 \mathrm{~mW} / \mathrm{cm}^{2}$.

Aujourd'hui, des essais cliniques sont en cours à l'Hôpital Universitaire de Saint Ignace à Bogota en Colombie sous la direction du Docteur Nathalie Charpak. Le produit a obtenu le marquage $\mathrm{CE}$ et est commercialisé (figure 4).

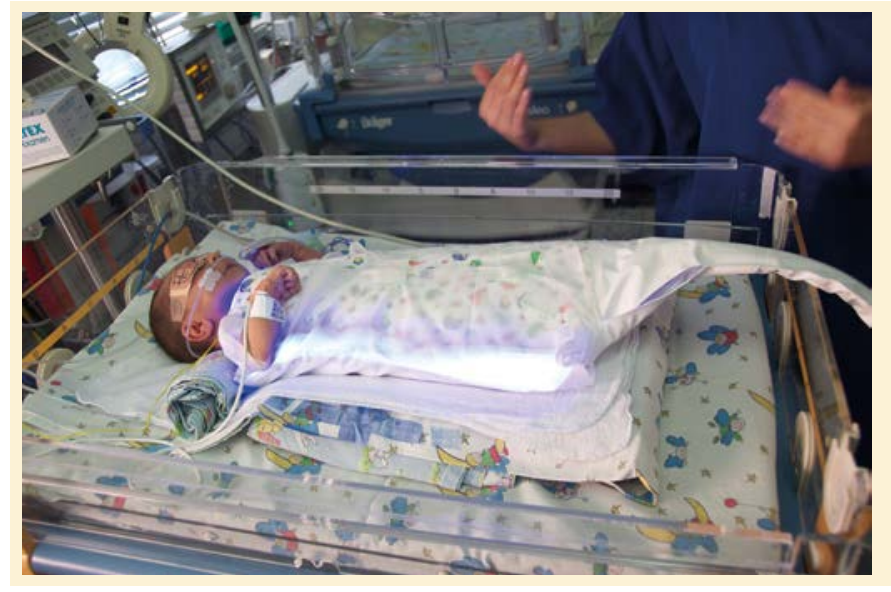

Figure 4. Essais cliniques à l'Hôpital Saint Ignace de Bogota (Colombie).

\section{RÉFÉRENCE}

[1] Henk Vreman, R.J. Wong, J.R. Murdoch, D.K. Stevenson, Standardized bench method for evaluting the efficacy of phototherapy devices, Acta Paediatrica 97, 308-316 (2008).

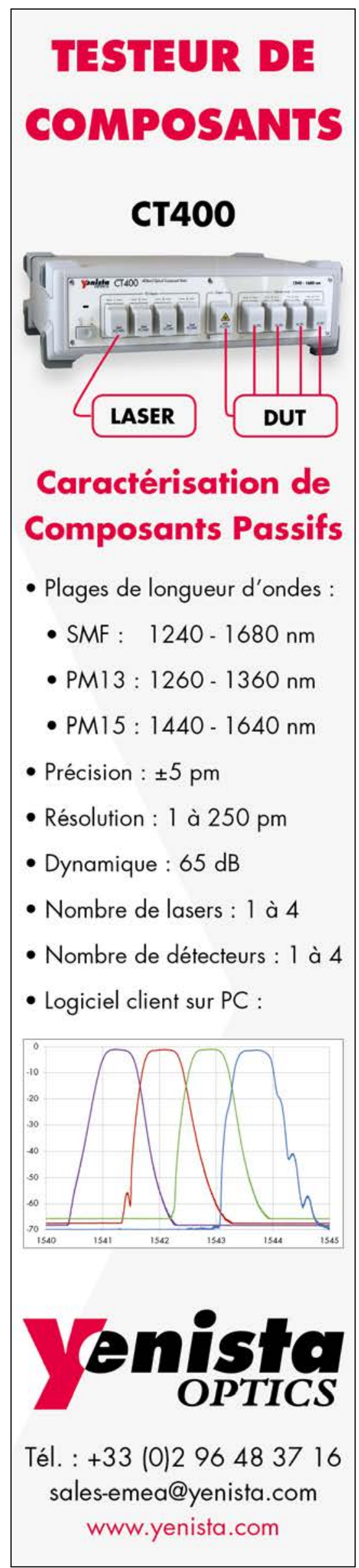

